\title{
El enfoque de la enseñanza directa y su impacto en la comprensión lectora de los alumnos de educación básica
}

\author{
Ángel Mizraim Canul Pool \\ angelcanul@crenjrg.edu.mx \\ Centro Regional de Educación Normal \\ "Javier Rojo Gómez" Unidad Cancún \\ Cancún - México
}

\section{RESUMEN}

En México, los bajos niveles de Comprensión Lectora señalados por distintas evaluaciones estandarizadas (SisAT, EXCALE, PLANEA-SEN y PISA), motivan esta investigación que plantea un análisis minucioso de las prácticas docentes cotidianas, para responder la pregunta de investigación: ¿Cómo el enfoque de la enseñanza directa en la alfabetización inicial impacta en la comprensión lectora de los alumnos de Educación Básica? Conocer esta interrelación contribuirá a resolver las problemáticas que aquejan la educación básica en nuestro país. El presente estudio considera los tres niveles de educación básica, preescolar, primaria y secundaria; a 190 docentes, es decir, el $70 \%$ de la población magisterial y a 5,171 alumnos, es decir, 73\% del total de adscritos a las diversas instituciones públicas y privadas de las zonas escolares 015 y 049 de Cancún, Quintana Roo. Para comprobar la hipótesis planteada y establecer la relación entre las variables, los datos recabados fueron sometidos a la prueba de chi-cuadrado $\left(x^{2}\right)$ en donde el resultado figuró como $12.9991>6.635$ por lo tanto (Hi) es positiva, de esta forma se precisa que el enfoque del método de adquisición de la lectoescritura empleado en la alfabetización inicial guarda estrecha relación y es determinante para la Comprensión Lectora.

Palabras clave: comprensión lectora; alfabetización inicial; educación básica; competencias comunicativas; lectoescritura. 


\title{
The direct teaching approach and its impact on the reading comprehension of basic education students
}

\begin{abstract}
The low levels of reading comprehension in Mexico indicated by different standardized assessments (SisAT, EXCALE, PLANEA-SEN and PISA) originate this research, which proposes a thorough analysis of daily teaching practices in order to answer the question "How does the direct teaching approach of initial literacy impact the reading comprehension of Basic Education students?" Knowing the answer to it will contribute to solve problems that afflict basic education in our country. The current study considers the three levels of basic education: preschool, primary and secondary. It involves 190 teachers, who represent $70 \%$ of the teaching population in zones 015 and 049 of Cancun Quintana Roo, and 5,171 students who represent $73 \%$ of the total number of students assigned to the different public and private institutions in the same school zone. To test the hypothesis and establish the relationship between the variables, the data collected was subjected to the chi-square test $\left(x^{2}\right)$ where the result was $12.9991>6.635$ therefore (Hi) is positive. This result shows that the focus of the literacy acquisition method used in initial literacy is closely related and is a determinant for reading comprehension skills.
\end{abstract}

Keywords: reading comprehension; initial literacy; basic education; communicative competencies; literacy.

Artículo recibido: 20. Julio. 2021 Aceptado para publicación: 18. Agosto. 2021 Correspondencia: angelcanul@ crenjrg.edu.mx Conflictos de Interés: Ninguna que declarar

\section{INTRODUCCIÓN}


En la actualidad, es imposible imaginar la vida cotidiana sin la lectura y la escritura, pese a esto es necesario enfrentar un cambio paradigmático, que parte de concebir la lectura como un proceso que no sólo conlleva la decodificación fonográfica de un código escrito, sino, como un proceso de significación y comprensión de la realidad que está interiorizada y que se vincula estrechamente con la escritura convirtiéndola así en un proceso; la lectoescritura. Al encontrarnos inmersos en este proceso es necesario considerar la comprensión lectora como un hecho consecuente y complejo, pues el simple acto sencillo de leer deja de ser inherente al de la decodificación y se transforma para atender las necesidades sociales que el lenguaje demanda.

La habilidad lectora constituye una de las bases de la educación formal y un instrumento fundamental para el aprendizaje a lo largo de la vida. En el ámbito educativo existe un consenso en que esta habilidad integra la comprensión, la reflexión y el empleo de los textos escritos para diversos propósitos, como son la adquisición de nuevos conocimientos, el desarrollo personal y la participación en la sociedad (INEE, 2013, p. $15)$.

A pesar de que el proceso de lectoescritura es fundamental para el desarrollo social del ser humano éste conlleva un reto significativo para su adquisición en la educación básica. Todos los niños aprenden en muy poco tiempo a hablar bien su lengua materna sin una enseñanza formal. Después, al poco tiempo llegan a la escuela y muchos parecen tener dificultades en el aprendizaje del lenguaje, especialmente del lenguaje escrito, su lectura y comprensión, esto a pesar de recibir la enseñanza de maestros diligentes que utilizan materiales a veces costosos y/o cuidadosamente elaborados. ¿Por qué la enseñanza informal en este ámbito sobresale ante la educación formal?, ¿cuáles son los retos que la educación básica presenta para el alumno?, ¿qué rol desarrollan los docentes en la adquisición de la lectoescritura?, ¿la naturaleza misma del lenguaje tendrá un papel significativo en la adquisición de la lectoescritura?, ¿por qué los alumnos que leen en muchas ocasiones no comprenden lo que leyeron?

Atendiendo la comprensión lectora como una necesidad, a partir del ciclo escolar 20132014, la Subsecretaría de Educación Básica (SEP), por medio de la Dirección General de Desarrollo de la Gestión Educativa puso a disposición de los supervisores y directores escolares el Sistema de Alerta Temprana (SisAT) que proporciona herramientas para la 
toma de lectura, la producción de textos escritos y el cálculo mental, conjunto de instrumentos sencillos que permiten recopilar y sistematizar información puntual y objetiva para sustentar las recomendaciones pedagógicas para dar certeza y primacía a los aprendizajes en la educación básica y la formación integral que esta población requiere, generar estrategias y condiciones para que estas instituciones sean el centro del trabajo académico del sistema educativo y gocen del respaldo necesario para cumplir su finalidad. La investigación se realizó con los docentes ubicados de las zonas escolares 015 y 049 de Cancún, Q. Roo, en el periodo comprendido de enero a marzo del ciclo escolar 20192020 y con información obtenida a través del SisAT tomando en cuenta únicamente los resultados generales obtenidos al concluir el ciclo escolar 2018-2019. En este contexto, los resultados promediales obtenidos por el SisAT en las zonas escolar 015 y 049, reflejan un panorama desalentador para la Educación Básica (Tabla 11).

Tabla 1.

Resultados promediales del SisAT al término de su aplicación en el Ciclo Escolar 2018-2019 en el área de la Comprensión Lectora

\begin{tabular}{lcccc}
\hline & Escuelas & Requiere apoyo & En desarrollo & Nivel esperado \\
\hline Preescolar & 9 & $32.9 \%$ & $15.1 \%$ & $2.8 \%$ \\
Primaria & 15 & $57.1 \%$ & $25.1 \%$ & $17.8 \%$ \\
Secundaria & 7 & $40.1 \%$ & $17.9 \%$ & $8.3 \%$ \\
\hline
\end{tabular}

Elaboración propia con información de la supervisión de las zonas escolares 015 y 049 de Cancún, Q. Roo.

Lo que significa que, a pesar de las actividades realizadas por las zonas escolares, la capacitación permanente a los docentes encargados de la adquisición de la lectoescritura, la profesionalización docente en el grado de estudios y las estrategias nacionales que se han vertido para atender la lectura y beneficiar la comprensión lectora, estas acciones no han sido suficientes.

\section{ESTRATEGIAS METODOLÓGICAS}

La presente investigación se aplicó bajo un enfoque cuantitativo donde se utiliza la recolección y análisis de datos para contestar la pregunta de investigación y el análisis estadístico, con el fin de establecer patrones de comportamiento en una población para probar la hipótesis con base en la interpretación estadística, de la misma forma se 
reconoce como estudio exploratorio ya que los antecedentes de estudios previos son escasos. Hernández, Fernández y Baptista (2014).

Continuando con el diseño metodológico, este estudio se define como no experimental, ya que no se busca manipular deliberadamente variables, sino que observa los fenómenos tal y como se dan en su contexto natural para después analizarlos (Hernández et al. 2014). Los participantes pertenecen a una misma muestra, pero constituyen dos grupos, a los cuales se observó para relacionar las variables propuestas y establecer una relación, al grupo 1, a los docentes, se les aplicó la técnica de investigación cuantitativa "encuesta" para determinar la variable independiente, mientras que al grupo 2, los alumnos, se consideraron los resultados obtenidos en la prueba estandarizada que propone el SisAT para conocer la variable independiente establecida.

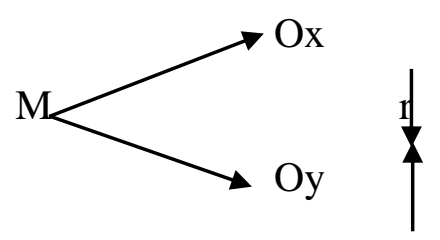

\section{En donde:}
$\mathrm{M}=$ Muestra .
Ox $=$ Observación de la variable independiente. Comprensión lectora.
Oy = Observación de la variable dependiente. Enfoque de alfabetización inicial.
$\mathrm{R}=$ Posible relación entre las variables del estudio.

\subsection{Población}

La población global está conformada por 259 docentes, y 6,840 alumnos de los niveles preescolar, primaria y secundaria adscritos a las zonas escolares 015 y 049 de Cancún, Quintana Roo, para este estudio no se consideran a los directivos ni a personal de apoyo escolar (PAE).

\subsubsection{Muestra}

Después de considerar la migración estudiantil y docente, y la ausencia de resultados en las pruebas SisAT para alumnos y la encuesta para docentes, se consideró a 190 profesores de educación básica y 5,040 alumnos que representan el 73.1\% de la población docente y estudiantil respectiva quedando como se describe a continuación:

\section{Tabla 2.}

Distribución de Muestra en las zonas escolares 015 y 049 de Cancún, Q. Roo.

Escuelas Docentes Alumnos Directores y PAE




\begin{tabular}{llccc}
\hline Preescolar & 8 & 22 & 633 & 27 \\
Primaria & 9 & 112 & 3016 & 45 \\
Secundaria & 7 & 56 & 1522 & 35 \\
\hline
\end{tabular}

Elaboración propia con información de la supervisión Escolar 015 y 049 de

Cancún, Q. Roo.

\subsection{Instrumento.}

Para obtener los datos del presente estudio se implementaron dos herramientas de investigación, la "encuesta" como instrumento para conocer el enfoque de alfabetización inicial empleado, traduciendo la concepción que se posee sobre la adquisición de la lectoescritura, esto impacta directamente en las estrategias y técnicas que los docentes de las zonas escolares 015 y 049 emplean para desarrollar la lectoescritura en sus alumnos; y los resultados promediales del SisAT al término de su aplicación en el Ciclo Escolar 2018-2019 en el área de la Comprensión Lectora. Los resultados sólo son válidos para expresar las opiniones en torno a las estrategias y conocimientos teóricos-metodológicos de la población objetivo en las fechas especificadas, la población de estudio fue determinada por exclusión, es decir aquellos docentes que no imparten estrategias para la adquisición de la lectoescritura, no fueron considerados, determinando el tamaño de la muestra 190 docentes, se estableció un nivel de confianza de 95\% con margen de error estadístico (190 encuestas) +/- 3.1\%. Además, puede haber errores no muestrales no considerados en la estimación. El método del muestreo fue sistemático y se llevó a cabo docente por docente de forma principalmente asincrónica y virtual a través de la herramienta digital Google Forms.

Dicha encuesta se aplicó del 16 al 21 de marzo del 2020, el instrumento fue facilitado a los docentes a través de los supervisores de cada nivel educativo con la autorización de la autoridad educativa en la zona norte de Quintana Roo.

Así mismo, como prueba referencial se consideraron los resultados obtenidos por el SisAT al final del ciclo escolar 2018-2019 en el área de Comprensión Lectora. Dicha prueba es un instrumento estandarizado que conlleva un conjunto de indicadores, herramientas y procedimientos que está diseñando para contar con información sistemática y oportuna acerca de los alumnos que están en riesgo de no alcanzar los aprendizajes esperados o de abandonar sus estudios (Tabla 11).

Con los resultados obtenidos se realizó un análisis para interpretar y correlacionar su 
dominio de los procesos de alfabetización inicial, su perspectiva, preferencia y conocimiento de los métodos para desarrollar la lectoescritura y finamente su conocimiento del desarrollo natural del niño desde la perspectiva de la neurolingüística con los resultados obtenidos en el primer momento del Ciclo Escolar 2018-2019 de la prueba SisAT con la intención de comprobar la hipótesis de esta investigación.

\subsubsection{Procedimiento para la obtención de datos.}

Para la obtención de los datos necesarios que sustentan la presente investigación fue necesario establecer el diálogo con distintas autoridades educativas y buscar su autorización, establecer un periodo para que los docentes accedan a la encuesta, determinar el momento para aplicar la prueba chi-cuadrado $\left(x^{2}\right)$.

\subsubsection{Procedimiento para la aplicación del tratamiento o estímulo.}

Para la presente investigación no se consideró la deliberada manipulación o tratamiento de las variables, lo que se buscó fue observar fenómenos tal y como se dan en su contexto natural, para después analizarlos. Como población del estudio se considera la totalidad de alumnos y docentes de educación básica, es decir preescolar, primaria y secundaria de las zonas escolares 015 y 049 de Cancún, Quintana Roo.

Después de valorar la migración estudiantil y docente, y la ausencia de resultados en las pruebas SisAT para alumnos y la encuesta para docentes, se consideraron dos muestras independientes dentro de la población:

El grupo 1, conformado por 190 profesores de educación básica, 70\% de la población docente, este grupo participó en una encuesta para conocer los métodos que emplean para desarrollar la lectoescritura en sus alumnos, y así determinar el enfoque utilizado, éste podría ser: de enseñanza directa, integral o constructivista.

Del 16 al 21 de marzo del 2020, se llevó a cabo la encuesta en sus modalidades digital e impresa en los centros educativo y fuera de ellos, cada docente determinó y gestionó el tiempo para ofrecer sus respuestas. El instrumento tuvo una duración máxima de 15 minutos por docente. Al finalizar la encuesta los docentes que decidieron responderla de manera impresa entregaron los formatos en fecha máxima el día 21 de marzo del presente año, los directores a su vez entregaron el día 23 de marzo a la supervisión escolar correspondiente.

Del grupo 2, conformado por 5,040 alumnos que representan el 73.1\% de la población estudiantil, se tomaron como referencia los datos obtenidos por el SisAT y 
proporcionados por la Supervisión Escolar de preescolar, primaria y secundaria en el ámbito de la comprensión lectora, al término del ciclo escolar 2018-2019.

El Sistema de Alerta Temprana (SisAT) es una herramienta que permite conocer el logro de las competencias en tres ámbitos distintos: pensamiento matemático, producción de textos escritos y comprensión lectora. Para esto se determinan dos periodos de aplicación, el primero funge como diagnóstico y se aplica en los primeros meses después de iniciado el ciclo escolar, esto permite a los docentes cambiar estrategias, técnicas o replantear su propia práctica pedagógica. El segundo periodo se aplica pocos meses antes de concluir el ciclo escolar y muestra el avance logrado respecto a su primera aplicación, en ambos casos el instrumento empleado es el mismo, a fin de garantizar un enfoque objetivo, cualitativo con diseño cuasiexperimental con preprueba y posprueba.

Al término de la aplicación de esta herramienta y durante el mes siguiente después del último momento de su aplicación, se realiza una recopilación de los resultados obtenidos a nivel de zona escolar, esto con la finalidad de conocer qué estrategias están funcionando y cuáles podrían implementarse en otras zonas escolares.

Para el ciclo escolar 2018-2019 el SisAT se llevó a cabo en el mes de marzo para la captura del primer momento y en el mes de octubre el segundo momento, sin embargo, durante el ciclo escolar 2019-2020 no se implementó esta herramienta debido a los últimos cambios en política educativa.

\section{RESULTADOS Y DISCUSIÓN}

Para llevar a cabo la comprobación de la hipótesis que presenta el este estudio, se utilizaron datos medibles en una escala nominal en donde la hipótesis nula de la evaluación Chi-cuadrado $\left(x^{2}\right)$. Sostiene una distribución de probabilidad totalmente especificada de acuerdo con el modelo matemático de la población utilizado en la muestra, es decir, para esta prueba serán: el número de docentes que emplean un enfoque directo en su práctica profesional para facilitar la lectoescritura con sus alumnos, y el número de alumnos que presentan dificultades en el área de la comprensión lectora, ambas muestras pertenecen a la población definida en las zonas escolares 015 y 049 de Cancún, Q. Roo, con la intención de comprobar la hipótesis:

Hi: El enfoque de la enseñanza directa en la alfabetización inicial influye en la comprensión lectora de los alumnos de Educación Básica de las zonas escolares 015 y 049 en Cancún Q. Roo. 
Ho: El enfoque de la enseñanza directa en la alfabetización inicial no influye en la comprensión lectora de los alumnos de Educación Básica de las zonas escolares 015 y 049 en Cancún Q. Roo.

\section{La hipótesis estadística:}

Donde: Hi: $r x y \neq 0$

$$
\text { Ho: } \operatorname{rxy}=0
$$

\section{Variables}

- Independiente: el enfoque de la enseñanza directa en la alfabetización inicial

- Dependiente: la comprensión lectora

\section{Unidades de análisis:}

190 profesores de Educación Básica (70\% de la población)

5,040 alumnos de Educación Básica (73.1\% de la población)

Para realizar este contraste se disponen los datos en una tabla de frecuencias. De acuerdo con Elsevier España S.L.U. (2021) para cada valor o intervalo de valores se indica la frecuencia absoluta observada (Oi). A continuación, y suponiendo que la hipótesis nula es cierta, se calculan para cada valor o intervalo de valores la frecuencia absoluta que cabría esperar o frecuencia esperada $(\mathrm{Ei}=\mathrm{n} \cdot \mathrm{pi}$, donde $\mathrm{n}$ es el tamaño de la muestra y pi la probabilidad del i-ésimo valor o intervalo de valores según la hipótesis nula). El estadístico de prueba se basa en las diferencias entre la Oi y Ei y se define como:

$$
x^{2}=\sum_{i=1}^{k} \frac{\left(O_{i}-E_{i}\right)^{2}}{E_{i}} \text {. }
$$

Partiendo del análisis de los ítems, las respuestas de los docentes y el resultado del SisAT obtenido al final del siclo escolar 2018-2019, se presenta la distribución para la prueba

\begin{tabular}{|c|c|c|c|}
\hline \multirow[t]{2}{*}{$\begin{array}{l}\text { Alumnos con resultados no } \\
\text { esperados en el ámbito de la } \\
\text { Comprensión Lectora }\end{array}$} & \multicolumn{2}{|c|}{$\begin{array}{c}\text { Enseñanza a través de métodos } \\
\text { sintéticos para fomentar la } \\
\text { adquisición de la } \\
\text { lectoescritura. }\end{array}$} & \\
\hline & Sí & No & \\
\hline Sí & 2967 & 1204 & 4171 \\
\hline No & 1114 & 604 & 1718 \\
\hline
\end{tabular}
Chi-cuadrado $\left(x^{2}\right)$. 


\begin{tabular}{|c|c|c|c|}
\hline Nivel de significancia 0.01 & 4081 & 1808 & \\
\hline
\end{tabular}

Figura X. Tabla de contingencia extendida: Valores o Frecuencias observadas (fo) Elaboración propia con información de Elsevier España S.L.U.

\begin{tabular}{|c|c|}
\hline 2890.44 & 1280.55 \\
\hline 1190.55 & 527.44 \\
\hline
\end{tabular}

Figura X. Valores o frecuencias esperadas $(f e)$

Elaboración propia con información de Elsevier España S.L.U.

\begin{tabular}{|c|c|}
\hline$\frac{(2967-2890.44)^{2}}{2890.44}=2.027$ & $\frac{(1204-1280.55)^{2}}{1280.55}=-0119$ \\
\hline$\frac{(1114-1190.55)^{2}}{1190.55}=-0.128$ & $\frac{(604-527.44)^{2}}{527.44}=11.112$ \\
\hline & $x^{2}=\sum \frac{(f o-f e)^{2}}{f e}=12.9991$ \\
\hline
\end{tabular}

Figura X. Cálculo de Chi-cuadrado $\left(\mathrm{x}^{2}\right)$.

Elaboración propia con información de Elsevier España S.L.U.

\begin{tabular}{|c|c|}
\hline Chi-cuadrado $\left(\mathrm{x}^{2}\right)$ Calculado & Chi-cuadrado $\left(\mathrm{x}^{2}\right)$ Crítico \\
\hline 12.9991 & 6.635 \\
\hline
\end{tabular}

Figura X. Comprobación de la hipótesis.

Elaboración propia con información de Elsevier España S.L.U.

Por lo tanto, la hipótesis no es nula, y se establece relación entre las variables previamente establecidas. La hipótesis Hi: $r x y \neq 0$ es correcta. Los métodos de enseñanza directa de la alfabetización inicial influyen en la comprensión lectora de los alumnos de las zonas escolares 015 y 049 de Cancún, Quintana Roo.

Este estudio se sustenta en las siguientes ideas de varios autores reconocidos en el tema. Sofía Vernon, (1996), menciona que existen tres distintos enfoques para la enseñanza inicial de la lengua escrita, mismos que guardan estrecha relación con el desarrollo de la comprensión lectora de acuerdo con S. Vosniadou (2000), del mismo modo, manifiesta que dos de los enfoques más conocidos y trabajados en este campo de la enseñanza son la enseñanza directa y el lenguaje integral. 
El enfoque denominado "Enseñanza directa" es universalmente el más difundido; se deriva de investigaciones agrupadas bajo el título de "Conciencia fonológica". Quienes apoyan esta postura suponen que el sistema alfabético de escritura es una transcripción de sonidos a letras y viceversa, por tanto, lo más importante a aprender es identificar esos sonidos y asociar cada uno con la letra correspondiente. Hacen énfasis en las habilidades de codificar y decodificar y el reconocimiento de palabras para poder leer. Insisten en que, para que esta identificación sea eficaz, es necesario que el niño desarrolle tales habilidades.

Quienes se oponen a la enseñanza directa, señalan que el dominio de estas habilidades fonológicas que sirven de base para el aprendizaje de la lectura y de la escritura es totalmente antinatural, porque no es parte de una situación comunicativa real. La habilidad de segmentar el lenguaje en sonidos (fonemas) no es lo esencial y hacerlo, lejos de favorecer el proceso de adquisición, lo obstaculiza; los programas de enseñanza de la lectura y la escritura son altamente estructurados, se enseñan de manera sistemática las destrezas complejas que intervienen en el proceso de adquisición de la lectura y la escritura y claramente se aprecia un modelo conductista en la enseñanza. Con estos métodos se aprende a leer de manera mecánica, lo que equivale a conceptualizar a la lectura como una simple decodificación grafofonética.

En resumen, bajo la enseñanza directa podemos agrupar distintos métodos para fomentar la adquisición de la lectoescritura el alfabético, el fonético, el silábico y finalmente el psicofonético, éstos se catalogan como sintéticos. Este enfoque se sustenta en un aprendizaje jerárquico, en el que las habilidades tienen un antecedente y un consecuente que permiten su desarrollo gradual, es decir, que pasa por momentos o etapas desde las más fáciles a las más difíciles. En este sentido, la enseñanza debe ajustar sus actividades didácticas a esta gradualidad. Este proceso sintético parte del estudio de las letras por separado, una por una (grafía o fonema), combinando con otra grafía o fonema para formar sílabas y luego con las sílabas formar palabras cada vez con mayor complejidad hasta formar enunciados y textos.

Kenneth y Yetta Goodman, proponen desde otra perspectiva, el enfoque del "Lenguaje integral". Argumentan que el aprendizaje de la lengua escrita es un aprendizaje "natural". Todos los niños aprenden a hablar sin que se les enseñe explícitamente. Aprenden porque interactúan con personas que usan su lengua para establecer comunicación entre ellas. 
Este aprendizaje se da de manera espontánea sin que nadie literalmente le enseñe a hablar. De igual manera, el niño que vive en un medio social letrado aprenderá a leer y escribir porque la necesidad de establecer comunicación lo obligará a participar de las convencionalidades de este medio de comunicación. Esto implica que el niño debe estar participando en un contexto en el cual la lengua escrita se use con propósitos comunicativos reales. Tal como Sofía Vernon menciona:

(...) Desde el inicio de su aprendizaje deben proporcionarse a los niños textos reales: cuentos, periódicos, propagandas, cartas, etc. En ningún momento deben usarse escritos realizados sólo para enseñar a leer, pues éstos usan un lenguaje artificial que nadie utiliza para comunicarse. (...) Debe evitarse la enseñanza directa de letras, sílabas, palabras y oraciones aisladas, ya que éstas se encuentran descontextualizadas y tienen poco sentido. Leer equivale a buscar significado, y éste se encuentra en los textos reales. Cualquier intento de simplificar el lenguaje y la estructura de un texto resultará en una violación que impedirá un aprendizaje real. (Vernon, 1996, p. 3)

Podemos ver en estos dos planteamientos, dos enfoques radicalmente opuestos: la comprensión contra la decodificación; la enseñanza directa contra el aprendizaje integral. El tercer enfoque es denominado "Constructivista". Tiene algunos puntos en común con el lenguaje integral, en particular, la noción de que leer y escribir parten de una situación comunicativa, y que los niños deben tener contacto con diferentes tipos de textos desde un inicio. Igualmente, señalan que leer no es decodificar, sino buscar significado. Este enfoque es el que permea los planes y programas 2017, y fue introducido inicialmente en 2011 al consolidar la RIEB. En las instituciones formadoras de docentes este cambio paradigmático se introdujo formalmente en 2012 con nuevos planes y programas. Sin embargo, una diferencia fundamental sitúa al enfoque de enseñanza integral acompañando el desarrollo de los futuros lectoescritores en cada aspecto de su vida y de acuerdo con su desarrollo, sin embargo, la enseñanza constructivista lo hace "hasta la edad escolar" y además esta última sitúa el logro de la lectura hasta el segundo ciclo de la educación primaria, es decir entre los 7-8 años según refiere el plan y programa 2027 "Aprendizajes clave para la educación integral".

\subsection{La comprensión lectura como consecuencia de la lectoescritura.}


Después de revisar distintos textos se define la comprensión lectora como la capacidad de un individuo para interpretar objetivamente la idea que el escritor pretende transmitir por medio del texto escrito. En este sentido, la comprensión lectora (Reading Comprehension) es un concepto asociado a la integración del texto a las estructuras del conocimiento del lector, más al hecho abstracto que depende de la capacidad de cada persona, más al individuo que al entorno y está subordinado a otro concepto más amplio, nos referimos a la competencia lectora (Reading Literacy). La competencia lectora consiste en la comprensión y el empleo de textos escritos, en la posibilidad de reflexión sobre los mismos y el empleo de los conceptos bajo una visión particular que permite el desarrollo del conocimiento, incluye un bagaje extenso de competencias de carácter cognitivo, características lingüísticas, hasta el conocimiento del mundo, implica el uso funcional del lenguaje escrito en situaciones objetivas, en este sentido, la competencia lectora es más pragmática y esta en relación directa con la sociedad, a su entorno donde se ejecuta o materializa. (Marina, 2012).

Al considerar el proceso de desarrollo de las capacidades cognitivas según la neurociencia que establece que “(...) El proceso de desarrollo cerebral es gradual y por ello las propuestas de aprendizaje deben ir de lo más simple y concreto a lo más abstracto y complejo y por ello las propuestas de aprendizaje deben ir de lo más simple y concreto a lo más abstracto y complejo." (Campos, 2010, p. 9) se vislumbra que el momento de mayor aprendizaje, es la primera infancia, es decir, el periodo de crecimiento desde el nacimiento hasta los 5 años según se define por la United Nations International Children's Emergency Fund (UNICEF) y de manera simultánea considerar el proceso de desarrollo de las competencias comunicativas señalado por Contreras (1999), quien citando a Chomsky, manifiesta que: “(...) Los humanos estamos genéticamente predispuestos para aprender la lengua oral a través del Mecanismo de Adquisición del Lenguaje (MAL) traduciéndolo en el innatismo"(p.16), es decir, el niño como agente activo en el aprendizaje de su lengua y a Bruner: el niño aprende a comunicarse al convivir con miembros de su cultura. Los modos de interacción en la infancia temprana constituyen rutinas que proporcionan un Sistema de Apoyo para la Adquisición del Lenguaje (SAAL), y finalmente conjugar que "a partir de los tres tipos de conocimiento que la componen, conocimiento para la interacción (I), conocimiento del mundo (M) y el conocimiento del código (C)" (Contreras, 1999, p. 17) de esta forma se infiere que las condiciones 
apropiadas para desarrollar las habilidades superiores inicia justamente en el nacimiento en un proceso gradual y decreciente hasta la edad adulta.

En conclusión, para favorecer el aprendizaje del lenguaje y en consecuencia la comprensión lectora es importante llevar a cabo una coordinación fina entre el proceso de desarrollo de las competencias comunicativas y el desarrollo de las capacidades cognitivas de acuerdo con los hallazgos de la neurociencia, mismo que debe responder a un enfoque integral para la alfabetización inicial sostenido por estrategias que respondan a las etapas naturales desarrollo del ser humano.

Estas afirmaciones al ser contrastadas con el análisis estadístico de la información obtenido a través de la encuesta se pueden apreciar que, el principal enfoque utilizado por los docentes en su práctica cotidiana es de "Enseñanza directa" se afirma esto con base en sus conocimientos teóricos-prácticos y su propia experiencia. Algunas de las aseveraciones más relevantes obtenidas en dicha encuesta fueron:

El 78\%, es decir 149 de los 190 profesores encuestados no encuentran relevancia entre el enfoque de enseñanza directa y el enfoque integral. Esto es significativo, ya que establece que los profesores imparten estrategias eclécticas no selectivas, del mimo modo se vislumbra el desconocimiento de las teorías que fundamentan los planes y programas actuales en el área de lenguaje y comunicación "Lengua Materna. Español”.

Por otra parte, el $31 \%$ de los profesores, es decir, 59 de los encuestados afirma que, para la adquisición de la lectoescritura, lo más importante es conocer el sonido de la letra o la sílaba, misma filosofía es atribuible a su práctica profesional. Misma cantidad (31\%) de docentes manifestó no estar a favor ni en contra de ningún método.

Del mismo modo, el 31\%, es decir, 49 profesores manifestaron que lo más importante es el contexto del texto en la adquisición de la lectoescritura, y 22\%, es decir, 42 de ellos considera que lo más importante es cuándo emplearlos, con esta afirmación están reconociendo la diversidad en el desarrollo de las competencias lingüísticas para partir de las individualidades y adaptar el método a las necesidades de los alumnos.

Finalmente, el 78\% de los profesores afirma que lo más importe es el dominio del método, con ello se pierde el enfoque centrado en alumnos y en sus procesos de aprendizaje plasmado en el primer principio pedagógico en el plan y programa 2017 y 2011 de educación básica y 2012 para las instituciones formadoras de docentes.

\section{CONCLUSIÓN}


Habiendo establecido la relación entre los métodos de enseñanza directa de la alfabetización inicial y su influencia en la comprensión lectora de los alumnos, además, considerando las nuevas evidencias que presenta la neuroeducación resulta necesario cambiar el concepto del aprendizaje del niño en consecuencia, el cambio paradigmático actual sobre la adquisición de la lectoescritura, para de esta manera, ofrecer una verdadera educación integral que no sólo beneficie el logro de las competencias comunicativas sino también otras áreas como el razonamiento lógico matemático, el aprendizaje de idiomas y las competencias lingüísticas.

En este cambio paradigmático la adquisición de las competencias comunicativas, la comprensión lectora, la expresión oral y la lectoescritura entre otras; no están seccionadas, sino que se entrelazan y desarrollan conjuntamente, por ello al referirnos a la comprensión lectora, es necesario dejar de buscar métodos y estrategias remediales para subsanar las carencias que el enfoque de enseñanza directa para la adquisición de le lectoescritura provoca a nivel neuronal.

Diversas investigaciones y estudios teóricos actuales sobre los procesos de lectura y escritura aportan consideraciones importantes que dan una visión nueva a estos procesos, desde su adquisición inicial hasta su dominio pleno. Sus conclusiones deben repercutir en la práctica educativa, generar mayor conciencia de las construcciones y dominios relevantes: a) enfatizar los antecedentes del proceso; b) considerar el desarrollo psicolingüístico y cognitivo alcanzado antes del inicio de la educación formal, por ejemplo, el desarrollo del lenguaje oral, de la conciencia alfabética y fonológica, y de los esquemas o estructuras de conocimiento sobre la realidad; y c) desarrollar niveles de conciencia o conceptualización del lenguaje escrito, por ejemplo la conciencia semántica y sintáctica. Si deseamos formar estudiantes con una mejor comprensión lectora, es vital que se atienda ésta desde la educación inicial a través de una metodología integral que provea lo necesario para dar el significado al lenguaje, generar hábitos y conexiones neuronales que favorezcan el sincretismo de las palabras, las oraciones y los textos.

El cuestionamiento continuo del niño sobre su entorno es una actividad central en su aprendizaje. Es esta curiosidad sobre el mundo que lo acerca a la comprensión de la lengua escrita, cuando reflexiona, formula hipótesis y se responde a sí mismo sobre los principios y reglas que rigen este medio de comunicación. La necesidad de comunicarse hace que finalmente descubra la naturaleza organizativa de la lengua escrita de manera 
espontánea, apropiándose de los signos lingüísticos y su competencia para usarlos en sus prácticas sociales de lenguaje de manera creativa.

El aprendizaje del lenguaje escrito debe permear con las emociones positivas para crear un aprendizaje significativo.

El procesamiento del lenguaje escrito está influenciado por el contexto. El conocimiento que tiene el sujeto del mundo, la cultura en que participa, las interacciones sociales en las que se desenvuelve, el contexto social y escolar con el que se relaciona y participa, entre otros ambientes donde socializa, son factores que condicionan la estructura mental del sujeto, mismas a las integra nueva información de manera constante. Por esto, es necesario conocer al alumno, los factores personales, sociales y ambientales que le permean antes de planear un proyecto con recursos teórico-metodológicos para la enseñanza formal. Permitir que los niños adquieran los conocimientos es muy importante; pero debe darse como un proceso natural, tanto en el hogar como en la escuela y debe estar presente en todas las actividades que el niño realiza en su vida diaria.

Propiciar la lectura con un enfoque funcional en la apropiación de la cultura, permite crear conciencia sobre el manejo cotidiano del lenguaje escrito. Aunado a esto, la institución educativa debe programar la enseñanza considerando el nivel conceptual alcanzado en el desarrollo espontáneo del niño y debe realizar actividades de enseñanza donde se estimule el autodescubrimiento.

De acuerdo con recientes investigaciones en el ámbito de la neurociencia y la linguística en referencia al lenguaje y el "signo lingüístico", ubican el inicio del proceso de adquisición de la lectoescritura, en la educación inicial, desde los 8 meses, momento en el que el desarrollo de la visión de profundidad y color culmina debido a la maduración de los conos y los bastones que son las células especializadas fotorreceptoras que se encuentran en la retina. De esta manera, la educación inicial manifiesta su importancia de la mano del método para así respetar siempre los procesos de crecimiento y desarrollo individual de cada infante.

Para concluir, se debe considerar la importancia del docente en el desarrollo de propuestas teórico-metodológicas sobre los procesos psicológicos y cognitivos que permean la práctica educativa de la lectura y la escritura en el marco de la neuroeducación para aportar a la adquisición y dominio de la lectoescritura. De igual forma, el docente debe tener en cuenta los avances científicos y tecnológicos, producto de la investigación en el 
campo educativo, que están influenciando nuevas perspectivas sobre el aprendizaje de la lectura y la escritura

\subsection{Recomendaciones.}

Con la finalidad de contribuir al Sistema Educativo Nacional en el ámbito de la Comprensión Lectora y la adquisición de la lectoescritura, tomando como base los resultados obtenidos en la investigación se realizan las siguientes sugerencias:

Incluir en la malla curricular de los programas que atienden la educación inicial, educación básica, las instituciones formadoras de docentes y otras con el mismo propósito, asignaturas destinadas al conocimiento de las neurociencias, la adquisición de la lecto escritura y su impacto en la comprensión lectora.

Implementar los métodos Doman, Philadelphia o Globales en las instituciones que atiendan la educación inicial (preescolar) y los métodos globales (sin descuidar la propuesta construcivista) en las instituciones de educación primaria con énfasis en el primer ciclo ( $1^{\mathrm{o}}$ y $2^{\mathrm{o}}$ grados), y finalmente los métodos sintéticos para los alumnos que no hayan adquirido la lectoescritura a partir del segundo ciclo de educación priamaria $\left(3^{\circ}\right.$, $4^{\circ}, 5^{\circ}$ y $6^{\circ}$ ) y en los casos excepcionales en la educación secundaria implementar programas complementarios con métodos globales de análisis estructural y los métodos sínteticos silábico-fonéticos.

Brindar capacitación permanente en metodología, estratégias, técnicas, materiales e instrumentos a los docentes encargados de dichos cursos y aquellos que enfrentan la necesidad de brindar lectoescritura en su aula.

\section{LISTA DE REFERENCIAS}

Amador, J. C. (2008). "La evaluación y el diseño de políticas educativas en México" Documento de trabajo No. 35, pp. 20.

Baez Pinal, G. E. (2009). Del catecismo a los libros de texto gratuitos. Un panorama histórico de la enseñanza del español en la escuela primaria. Historia y presente de la enseñanza del español en México. Coord. José G. Moreno de Alba. México: UNAM, pp. 13-186

Baez Pinal, G. E. y A. V. Canizal Arévalo (2009). El español en la escuela secundaria: un panorama histórico (1926-1993). Historia y presente de la enseñanza del 
español en México. Coord. José G. Moreno de Alba. México: UNAM, pp. 235369

Barbosa Held, Antonio (1983). ¿Cómo han aprendido a leer y escribir los mexicanos? México: Editorial Pax-México (1ª edición, 1971).

Bazant, M. (2005). Lecturas del Porfiriato. Historia de la lectura en México. Seminario de Historia de la Educación en México. México: COLMEX, pp. 205-242

Cabrol, M. y Székely, M. (eds.) (2012). Educación para la transformación, México: Banco Interamericano de Desarrollo.

Cámara de diputados. (2012). Pacto por México.

Caminal, M. (ed.) (2010). Manual de Ciencia Política, Madrid, ES: Editorial Tecnos.

Castillo, B. A. (2005). La escuela racionalista de Yucatán: una experiencia mexicana de educación anarquista, 1915-1923.

Del Castillo-alemán, G. (2012). "Las políticas educativas en México desde una perspectiva de política pública: gobernabilidad y gobernanza”, en Magis, Revista Internacional de Investigación en Educación, 4(9), 637-652.

Diario Oficial de la Federación (2013a). "Decreto por el que se reforman, adicionan y derogan diversas disposiciones de la Ley General de Educación”. Recuperado el 25 de enero de 2016, de: Recuperado el 25 de enero de 2016.

Diario Oficial de la Federación (2013b). "Plan Nacional de Desarrollo 2013-2018", Recuperado el 20 de enero de 2016, de: "Plan Nacional de Desarrollo 2013-2018", Recuperado el 20 de enero de 2016.

Diario Oficial de la Federación (2013c). "Programa Sectorial de Educación 2013-2018", Recuperado el 12 de marzo de 2016, de: "Programa Sectorial de Educación 2013$2018 \%$.

Ferreiro, Emilia (2013). El ingreso a la escritura y a las culturas de lo escrito. Textos de investigación, México: Siglo XXI.

Freire, P. (2005). Pedagogía del oprimido, Ciudad de México: Siglo XXI.

Freire, P. (2012). Pedagogía de la indignación. Cartas pedagógicas en un mundo revuelto, Buenos Aires, AR: Siglo XXI.

Galtung, J. (2004). Trascender y transformar. Una introducción al trabajo de los conflictos, México: Transcend/UNAM. 
Gómez, M. (2012). La Tutoría Académica en la Facultad de Ciencias Políticas y Sociales de la Universidad Autónoma del Estado de México desde la perspectiva de los Estudios para la Paz, México: Porrúa.

Gómez, M. (2015). Análisis de las políticas gubernamentales en México sobre Paz y Educación 2013-2018, Revista de Humanidades [en línea], 24, artículo 3.

Greaves, C. (2005). La Secretaría de Educación Pública y la lectura, 1960-1985. Historia de la lectura en México. Seminario de Historia de la Educación en México. México: COLMEX, pp. 338-372

Huerta Mendoza, G (1982). Los libros de Lengua Nacional y los Programas de 1957 y 1960. En: González Pedrero, E. Los libros de texto gratuitos. México: CONALITEG, pp. 99-114

Instituto Nacional de Evaluación Educativa. (2004), "Resultados de las pruebas PISA 2000 y 2003 en México".

Instituto Nacional de Evaluación Educativa. (2015). ¿Qué es la prueba PISA? Recuperado el 08 de febrero de 2016 de: Recuperado el 08 de febrero de 2016.

Instituto Nacional para la Evaluación de la Educación (2013), La expresión escrita en alumnos de primaria, Materiales para apoyar la práctica educativa, México, 2008. Investigación no experimental. (2012, marzo 30). EcuRed, . Consultado el 19:48, julio 25, 2021. en https://www.ecured.cu/index.php?title=Investigaci\%C3\%B3n_no $\underline{\text { experimental\&oldid }=1451545}$

Ischinger, B. (2010). Implementación de política educativa. Recuperado el 26 de febrero de 2016.

Jares, X. (1999). Educación para la Paz. Su teoría y práctica, Madrid, ES: Editorial Popular.

Jares, X. (2004). Educar para la paz en tiempos difíciles. Bilbao, ES: Bakeaz.

Juárez, Erik. (2016). Educación futura. Recuperado el 24 de enero de 2017 de: Recuperado el 24 de enero de 2017.

Loyo, E. (2005). La lectura en México, 1920-1940. Historia de la lectura en México. Seminario de Historia de la Educación en México. México: COLMEX, pp. 243294.

Martínez, F. (2001). Las políticas educativas mexicanas antes y después de 2001, Revista Iberoamericana de Educación, 27. 
Merino, M. (2013). Políticas Públicas, Ensayo sobre la intervención del Estado en la solución de problemas públicos. México: CIDE.

Nieto, R. (2014). Las políticas educativas en la Educación en México. Recuperado el 15 de febrero de 2016.

Oliveros, J. J. L., \& Valle, J. A. (1991). Enseñanza de la lectura y psicología: análisis de los" métodos" sintéticos. Psicothema, 3(1), 121-136.

Organización de las Naciones Unidas. (1999). Asamblea General. Resolución A/RES/53/243 Declaración y Programa de acción sobre una Cultura de paz

Organización para la Cooperación y el Desarrollo Económicos (2015). Programa Internacional de Evaluación de los Alumnos (PISA).

Organización para la Cooperación y el Desarrollo Económicos. (2015).

Pardo, M. C. (2004). De la Administración Pública a la Gobernanza, México: El Colegio de México.

Parsons, W. (2007). Políticas Públicas. Una introducción a la teoría y práctica del análisis de políticas públicas. México: FLACSO.

Rincón, C. F. C., \& Comunitario, C. S. (2009). Competencias Comunicativas. Escuela de Ciencias Sociales, Artes y Humanidades

Rodríguez, J. (2001). Reforma administrativa y nuevas políticas públicas, México: Editorial Novum.

Secretaría de Educación Pública, Plan y programas de estudio 2011. Educación Básica, México, 2011.

Secretaría de Educación Pública. (2013). Portal de la Secretaría de Educación Pública.

Secretaría de Educación Pública. (2015). Reformas educativas recientes y nueva Ley de Educación. La modernización educativa en México. Centro de Investigación y Estudios Avanzados del IPN, INEA-SEP.

SEP, Aprendizajes clave para la educación integral, Lengua Materna. Español. Educación secundaria. Plan y programas de estudio, orientaciones didácticas y sugerencias de evaluación, México, 2017.

SEP, Plan y programas de estudio 2011. Educación Básica, México, 2011.

Triglia, A. (2019). Las 4 etapas del desarrollo cognitivo de Jean Piaget.

Tuvilla, J. (2004). Cultura de paz y educación, en Molina, B. y Muñoz, F. (Eds.), Manual de Paz y Conflictos (pp. 387-425), Granada, ES: Universidad de Granada. 
Vernon, S. (1996). Tres distintos enfoques en las propuestas de alfabetización inicial. Básica, Revista de la escuela y el maestro, 9, 63-71. 